\title{
Topographische Untersuchung über die Abgangsstelle der grossen Bauchaortenäste bei den japanischen Kindern.
}

\author{
Von \\ Masahisa Endo. \\ Aus dem Anatomischen Institut der Keio Universität, Tokyo. \\ Mit 6 Textfiguren. \\ Inhalt. \\ I. Einleitung . . . . . . . . . . . . . . . 395 \\ II. Material und Methode . . . . . . . . . . . . . . 395 \\ III. Eigene Befunde . . . . . . . . . . . . . . . . . . . . 396 \\ IV. Zusammenfassung . . . . . . . . . . . . . . . . . 406 \\ Literatur . . . . . . . . . . . . . . . . 407
}

\section{Einleitung.}

Über das Lageverhältnis der grossen Äste der Bauchaorta gegen die Wirbelsäule bei den Japanern haben viele Autoren, wie B. Adachi (1928), N. Tsuka moto (1929), T. Taniguchi (1931) u.a. schon eingehend berichtet; aber gibt es keine Ergebnisse über die Kinder und Feten. Ich will deshalb hier über meine Befunde bei den japanischen Kindern hauptsächlich in Bezug auf Verhältnisse der Alterverschiedenheit berichten.

\section{Material und Methode.}

Die Untersuchungen wurden an Leichen von Kindern, Säuglingen und Neugeborenen, wie die Tabelle I zeigt, durchgeführt. In dieser Abhandlung habe ich nach B. A dachi die Mittelwerte der Ursprungshöhe (durchschnittliche oder mittlere Ursprungshöhe) errechnet. Dabei behandelte ich die einzelnen Drittel der Wirbelkörper und die Zwischenscheibe als gleichweite 
Tabelle I.

\begin{tabular}{|c|c|c|c|}
\hline \multirow{3}{*}{ Alter } & \multicolumn{3}{|c|}{ Zahl der Fälle } \\
\hline & \multicolumn{2}{|c|}{ Geschlecht } & \multirow{2}{*}{ Summe } \\
\hline & $\widehat{\delta}$ & 우 & \\
\hline o- I m & 3 & 4 & 7 \\
\hline $\mathrm{I}-2 \mathrm{~m}$ & 3 & 4 & 7 \\
\hline $2-3 \mathrm{~m}$ & 4 & 2 & 6 \\
\hline $3-4 \mathrm{~m}$ & 3 & 4 & 7 \\
\hline $4-5 \mathrm{~m}$ & 5 & 3 & 8 \\
\hline $5-6 \mathrm{~m}$ & o & 3 & 3 \\
\hline $6-7 \mathrm{~m}$ & 5 & I & 6 \\
\hline $7-8 m$ & $x$ & 2 & 3 \\
\hline $8-9 \mathrm{~m}$ & 3 & $\mathbf{I}$ & 4 \\
\hline $9^{--r o m}$ & 4 & o & 4 \\
\hline $10-11 \mathrm{~m}$ & $\circ$ & 2 & 2 \\
\hline $\mathrm{II}-\mathrm{I} 2 \mathrm{~m}$ & 2 & 2 & 4 \\
\hline$I^{-}-2 j$ & 18 & I6 & 34 \\
\hline $2-3 i$ & 3 & 3 & 6 \\
\hline $3-4 j$ & 3 & 3 & 6 \\
\hline $4-5 j$ & 0 & I & $\mathbf{I}$ \\
\hline $5-6 i$ & I & $\mathrm{I}$ & 2 \\
\hline $6-7 \dot{j}$ & I & o & I \\
\hline $7-8 j$ & $\mathbf{I}$ & $\mathbf{r}$ & 2 \\
\hline $8-9 j$ & I & o & I \\
\hline $9-101$ & o & 0 & $\circ$ \\
\hline IO-II j & I & $\circ$ & $\mathbf{I}$ \\
\hline$I I-12 j$ & o & o & $\circ$ \\
\hline $12-13 j$ & $\circ$ & $\circ$ & o \\
\hline $13-141$ & I & $\circ$ & I \\
\hline Summe & 63 & 53 & II 6 \\
\hline
\end{tabular}

Klassen der Reihenvariation; die Klassengrenzen wurden vom unteren Drittel des XI. Brustwirbels durch $\mathrm{x}, 2,3, \ldots$ gezeichnet, so zeigt z.B. die Zahl zwischen 2 und 3 das obere Drittel des XII. Brustwirbels.

Ich habe alle Fälle (116) in 6 Gruppen nach dem Alter, wie die Tabelle 2 zeigt, unterschieden. Die

Tabelle 2 .

\begin{tabular}{|c|c|c|}
\hline Gruppe & Alter & Zahl \\
\hline I & O- $-6 \mathrm{~m}$ & 38 \\
2 & $6-12 \mathrm{~m}$ & 23 \\
3 & $\mathrm{I}-2 \mathrm{j}$ & 34 \\
4 & $2-4 \mathrm{j}$ & $\mathrm{I} 2$ \\
5 & $4-9 \mathrm{j}$ & 7 \\
6 & $9-14 \mathrm{j}$ & 2 \\
\hline
\end{tabular}

5. Gruppe und 6. Gruppe wurden wegen ihrer wenigén Fallzahl in der statistischen Beobachtung nicht berücksichtigt.

\section{Eigene Befunde.}

\section{I) A. coeliaca.}

Die A. coeliaca entspringt bei meinen Fällen in dem Gebiet vom kaudalen Drittel des XI. Brustwirbels bis zum mittleren Drittel des I. Lendenwirbels und geht am häufigsten in der Höhe des mittleren Drittels des XII. Brustwirbels ab (Tabelle 3). Der Mittelwert der Ursprungshöhe in jeder Altersstufe ist 3.3 bei der 1. Gruppe (0-6 monatig), 3.4 bei der 2. Gruppe (6-1 2 monatig), 3.7 bei der 3. Gruppe (I-2 jährig) und 3.8 bei der 4 . Gruppe (2-4 jährig). Die Abgangsstelle dieser Arterie zeigt mit zunehmendem Alter eine allmählige Absteigerung.

Vergleicht man meine Befunde mit denjenigen der Neugeborenen (Reicher) und Erwachsenen (die Befunde von Adachi, Heidsieck, Tsukamoto, Taniguchi, Reicher und Anson-McVay zusammen- 
Tabelle 3. A. coeliaca.

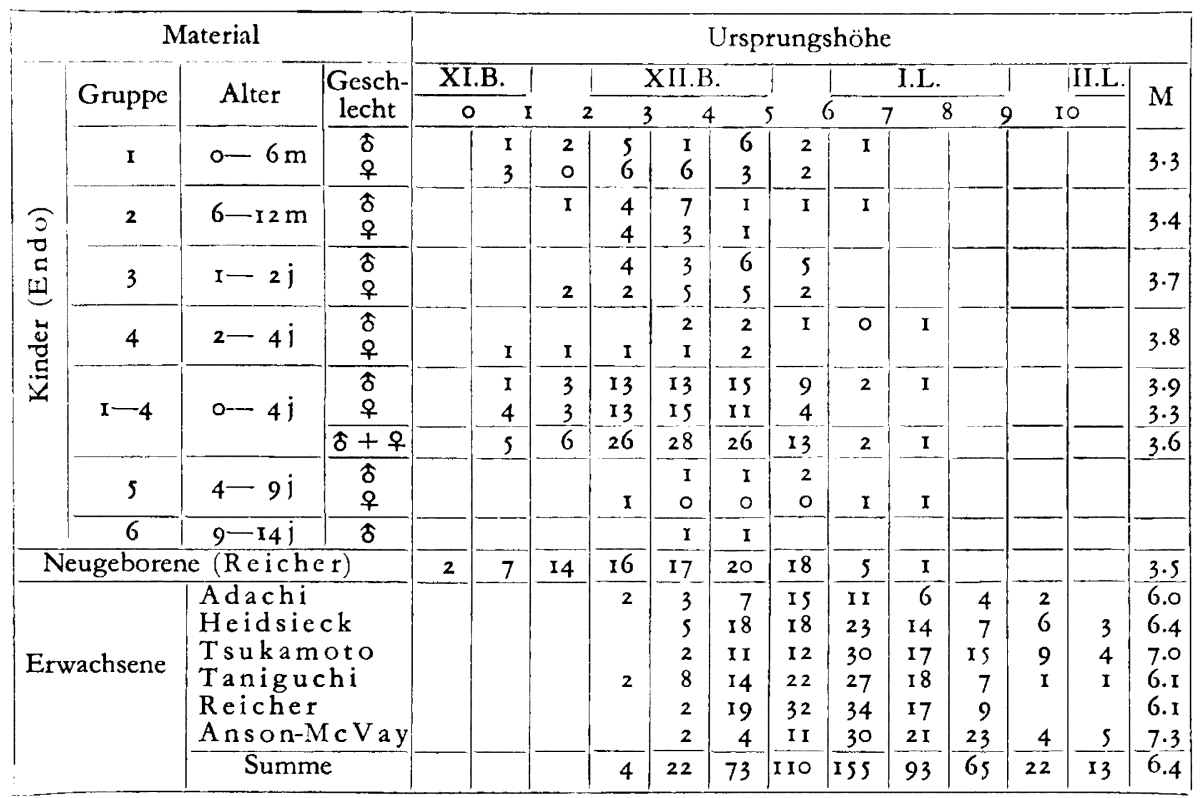

$\mathrm{M}=$ Mittelwert der Ursprungshöhe, $\mathrm{B}=$ Brustwirbel, $\mathrm{L}=$ Lendenwirbel.

gestellt), so liegt die Abgangsstelle bei Kindern etwas niedriger als bei Neugeborenen und ist bei Erwachsenen beträchtlich niedriger, fast um die Höhe eines Wirbelkörpers als bei Kindern, wie die Figur I zeigt.

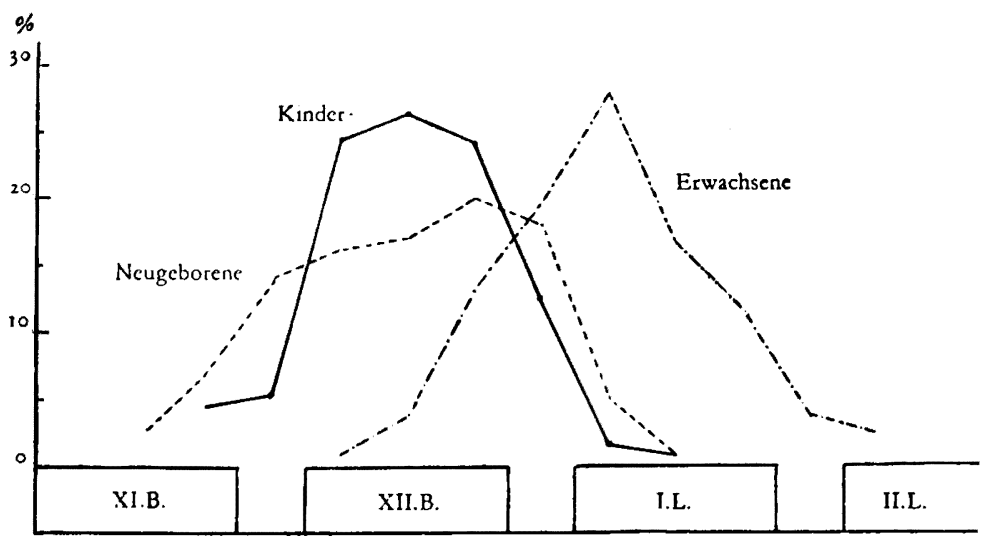

Fig. I. A. coeliaca.

Dem Geschlechte nach ist die Ursprungsstelle bei männlichen Kindern (M (Mittelwert der Ursprungshöhe) $=3.9)$ niedriger als bei weiblichen $(\mathrm{M}=$ 3.3). 


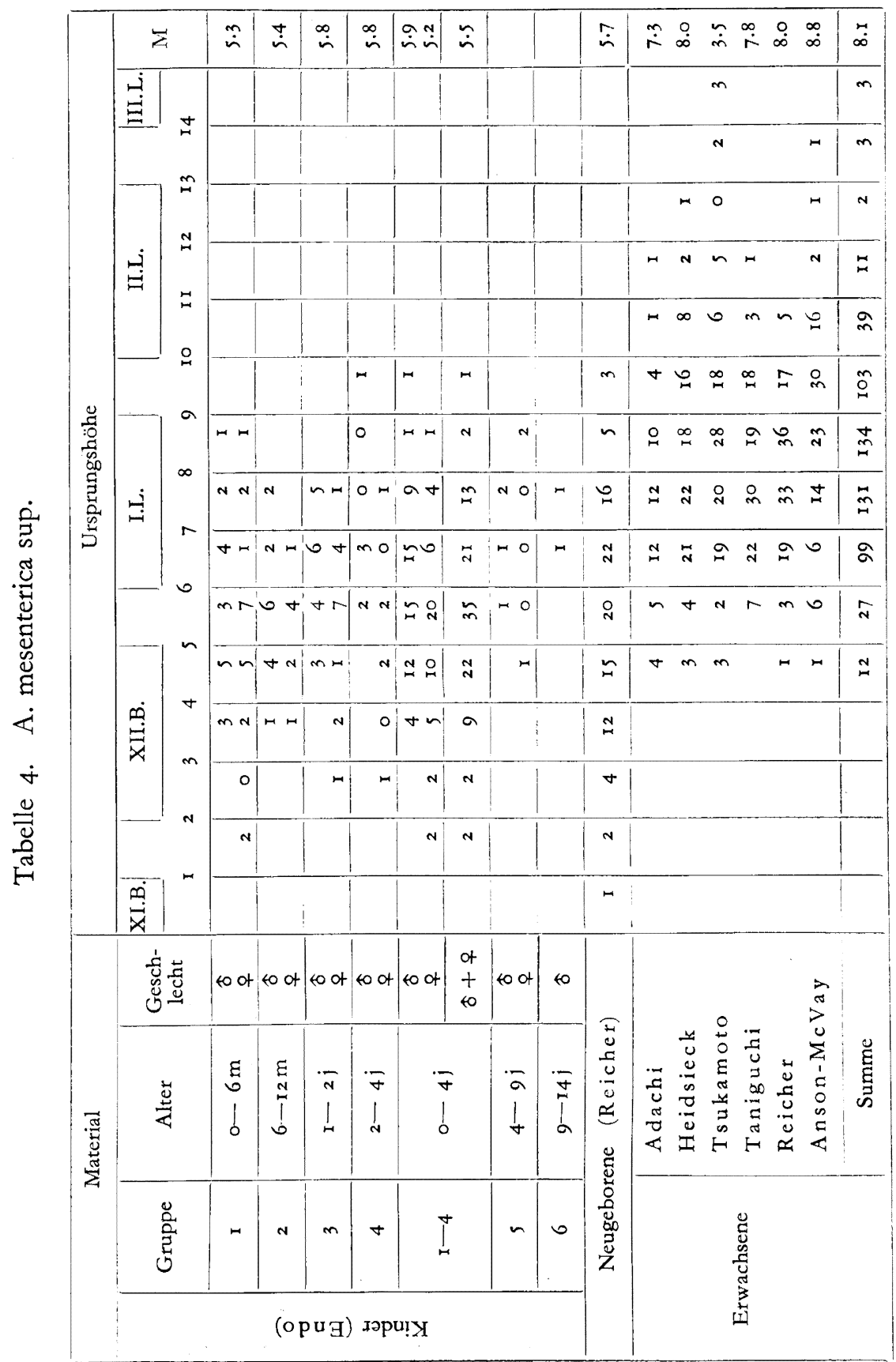




\section{2) A. mesenterica sup.}

Die Ursprünge dieser Arterie breiten sich bei meinen Fällen über das Gebiet von der Bandscheibe zwischen dem XI. und XII. Brustwirbel bis zu der Bandscheibe zwischen dem I. und II. Lendenwirbel, am häufigsten geht sie in der Höhe der Bandscheibe zwischen dem XII. Brust- und I. Lendenwirbel ab. Der Mittelwert der Ursprungshöhe in jeder Altersstufe zeigt 5.3 bei der I. Gruppe, 5.4 bei der 2. Gruppe, 5.8 bei der 3. und 4. Gruppe. Auch steigt dieser Ursprung mit zunehmendem Alter allmählich ab. Wie die Tabelle 4, welche die Resultate von Neugeborenen (Reicher) und Erwachsenen (Adachi, Heidsieck, Tsukamoto, Taniguchi, Reicher und Anson-McVay) enthält, erhellt, liegt der Mittelwert bei Neugeborenen in der Nähe meiner 3. und 4. Gruppe; bei Erwachsenen ist die Abgangsstelle beträchtlich niedriger als bei Neugeborenen und Kindern (Fig. 2).

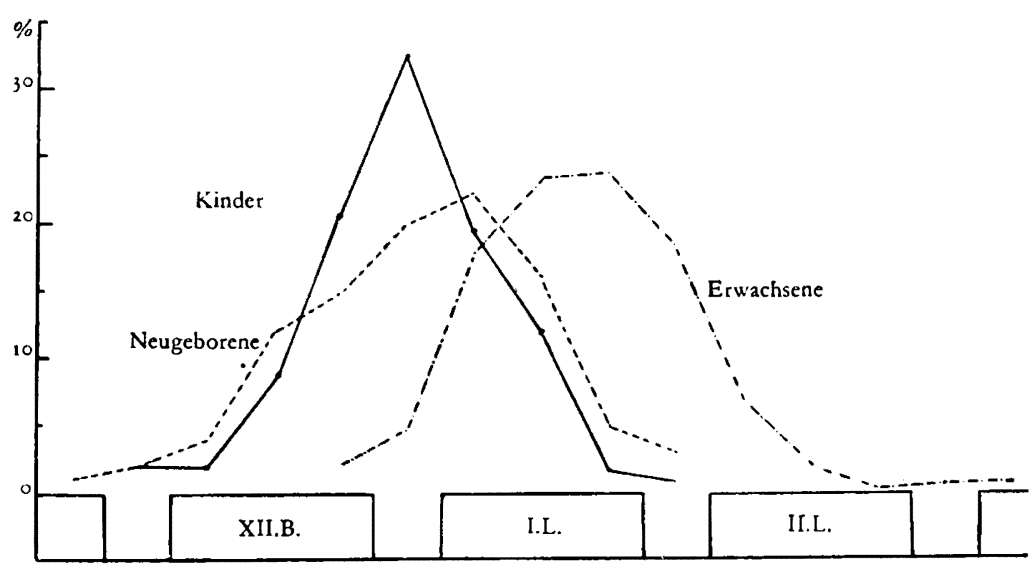

Fig. 2. A. mesenterica sup.

Dem Geschlecht nach ist die Abgangsstelle im allgemeinen bei männlichen Kindern $(M=5.9)$ niedriger als bei weiblichen $(M=5.2)$.

\section{3) Aa. renales.}

(a) A. renalis dextra.

Diese Arterien gehen bei mir in dem Gebiete vom kaudalen Drittel des XII. Brustwirbels bis zu dem mittleren Drittel des II. Lendenwirbels ab und entspringen am häufigsten im mittleren Drittel des I. Lendenwirbels. Die Mittelwerte der Ursprungshöhe in jeder Altersstufe sind 7.4 bei der I., 7.2 bei der 2., 7.5 bei der 3. und 7.4 bei der 4. Gruppe. Bei der 2. Gruppe ist 


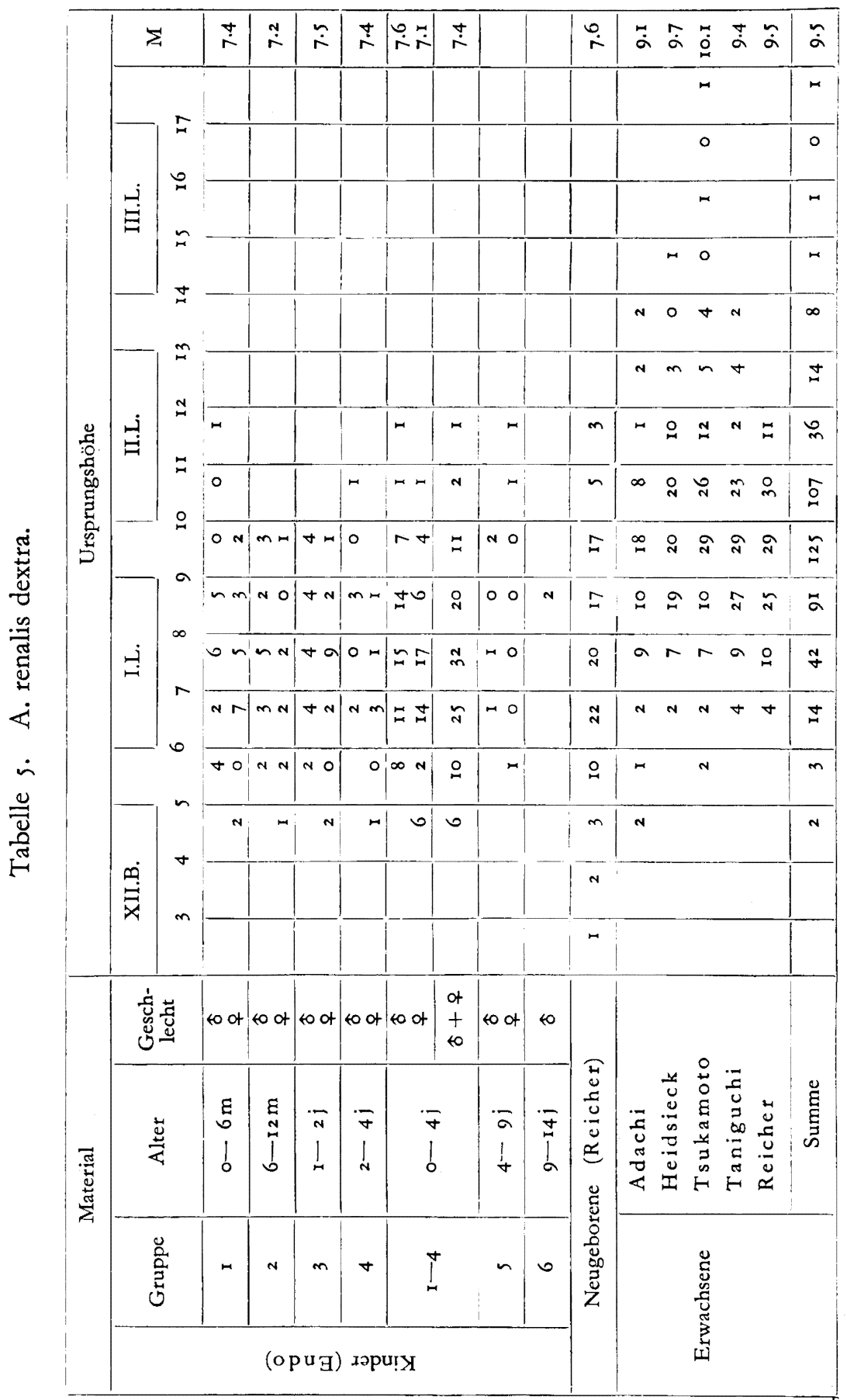


ihre Ursprungsstelle am höchsten, bei der 3. Gruppe am niedrigsten, und bei der I. und 4. Gruppe in der gleichen Höhe gelegen. Vergleicht man den Mittelwert der Ursprungshöhe bei Kindern mit dem bei Neugeborenen und bei Erwachsenen, so ist er, wie die Tabelle 5 angibt, etwas kleiner als bei Neugeborenen, und bei Erwachsenen beträchtlich größer als bei meinen Fällen. Die Abgangsstelle ist bei Kindern fast um zwei Drittel eines Wirbelkörpers höher als bei Erwachsenen, wie aus der Figur 3 ersichtlich ist.

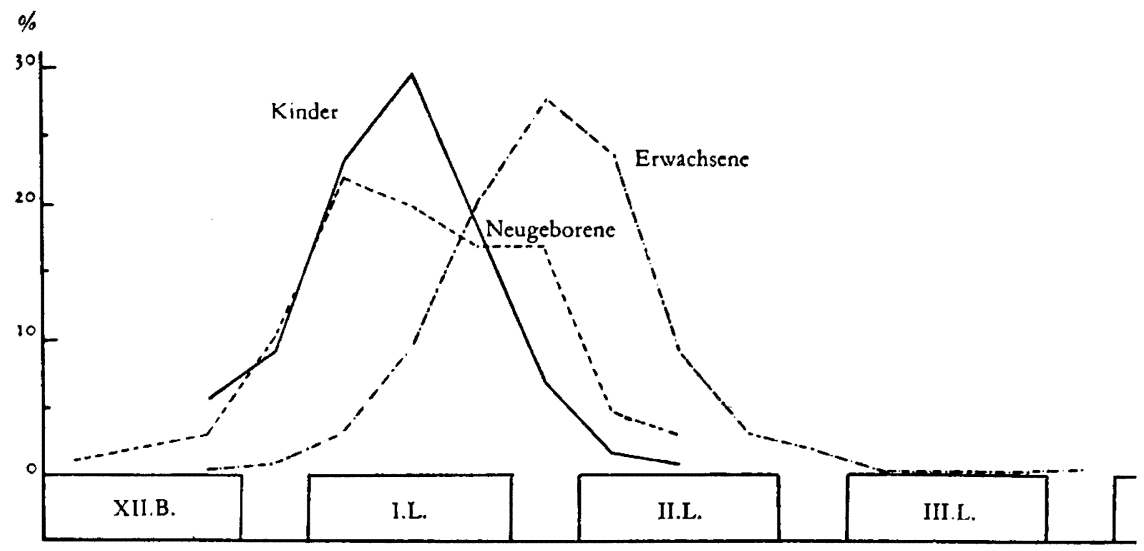

Fig. 3. A. renalis dextra.

Dem Geschlechte nach ist die Ursprungshöhe im allgemeinen bei männlichen Kindern $(M=7.6)$ niedriger als bei weiblichen $(M=7.1)$.

\section{(b) A. renalis sinistra.}

Diese Arterien entspringen bei mir in dem Gebiete vom kaudalen Drittel des XII. Brustwirbels bis zu dem kranialen Drittel des II. Lendenwirbels und gehen am häufigsten in der Höhe des kaudalen Drittels des I. Lendenwirbels ab. Ich habe als Mittelwerte der Ursprungshöhe in jeder Altersstufe 7.6 bei der 1. Gruppe, 7.5 bei der 2. Gruppe, 8.2 bei der 3. Gruppe und 7.6 bei der 4 . Gruppe erhalten, wie die Tabelle 6 zeigt. Nämlich sie zweigt sich bei der 2 . Gruppe am höchsten, bei der 3. Gruppe am niedrigsten, und bei der I. und 4 . Gruppe fast in der gleichen Höhe ab. Beim Vergleich der Mittelwerte der Ursprungshöhe bei Kindern mit dem bei Neugeborenen und Erwachsenen findet man, dass der Ursprung bei Neugeborenen ein wenig und bei Erwachsenen ziemlich niedriger als bei meinem Material liegt. Aus der Figur 4 ersieht man, dass der Ursprung bei Erwachsenen fast um die Hälfte der Höhe eines Wirbelkörpers niedriger als bei Kindern liegt. 


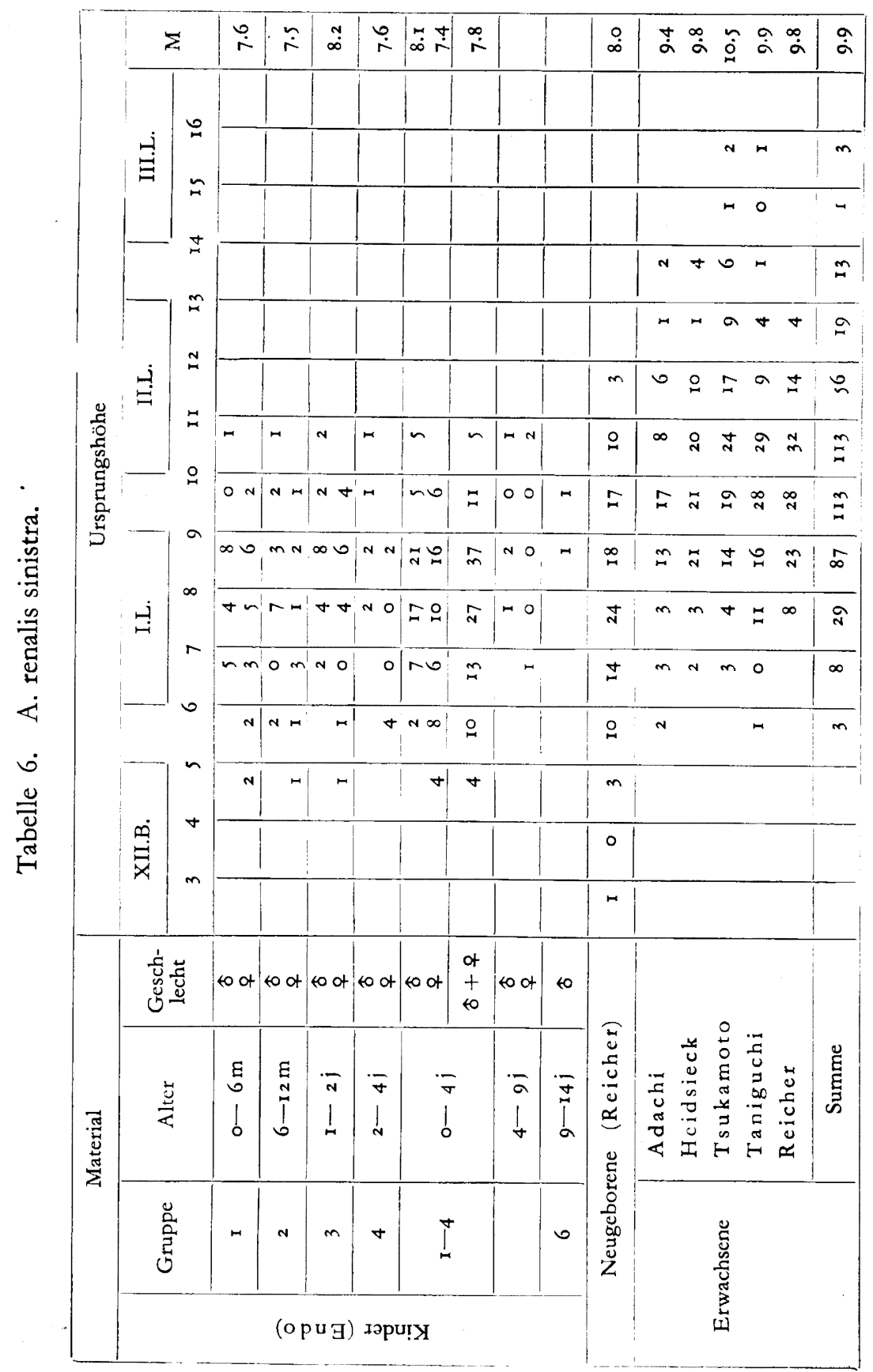




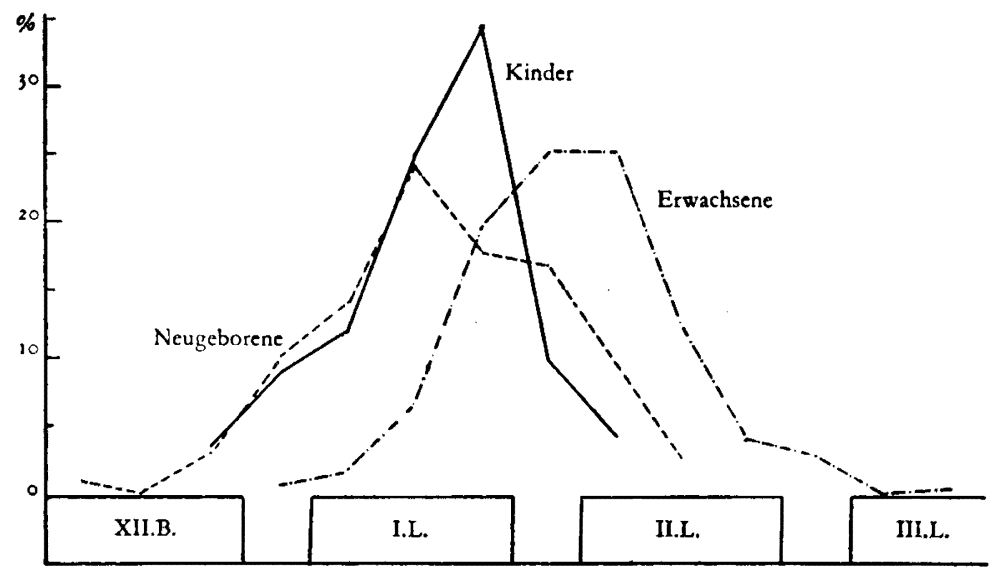

Fig. 4. A. renalis sinistra.

Bezüglich des Geschlechtes fand ich eine höhere Abzweigung bei weiblichen Kindern $(M=7.4)$ als bei den männlichen $(M=8.1)$.

(c) Ursprungshöhe der beiderseitigen Aa. renales.

Es ist allbekannt, dass die rechte und linke Nierenarterie oft nicht in gleicher Höhe entspringen. Ich fand bei 33 unter 1 I 6 Fällen, also bei $28.45 \%$, den Ursprung beiderseits gleich hoch, in 83 Fällen, also bei $71.55 \%$, dagegen ungleich hoch, und zwar 57 mal links kaudaler als rechts und 26 mal rechts kaudaler als links. Also entspringt die A. renalis dextra bei meinem Material höher als die A. renalis sinistra.

Wie aus der Tabelle 7 ersichtlich ist, stimmen meine Befunde mit den Befunden der bisherigen Autoren vollständig überein. So kann man mit Sicherheit schliessen, dass die rechte NierenTabelle 7. Vergleich der Ursprungshöhe der beiderseitigen Aa. renales. arterie auch im Kindes-

\begin{tabular}{|l|c|c|c|}
\hline \multicolumn{1}{|c|}{ Autor } & rechts höher & links höher & $\begin{array}{c}\text { beiderseits } \\
\text { gleich hoch }\end{array}$ \\
\hline Endo & 57 & 26 & 33 \\
Adachi & 69 & 20 & 16 \\
Heidsieck & 25 & 14 & 43 \\
Levi & 5 & 15 & 35 \\
Kubo & 8 & 6 & 6 \\
Iijima & 10 & 6 & I \\
Nagasawa & 53 & 27 & 15 \\
Ooniwa & 23 & 3 & 4 \\
Tsukamoto & 24 & 7 & 31 \\
Taniguchi & 47 & 21 & 32 \\
A so & 28 & II & 9 \\
Reicher & 97 & $5 \mathrm{I}$ & 37 \\
\hline \multicolumn{1}{|c|}{ Summe } & 442 & 207 & 262 \\
\hline
\end{tabular}
alter wie bei Erwachsenen gewöhnlich höher als die linke entspringt. 


\section{4) A. mesenterica inf.}

Der Ursprung findet sich bei meinen Fällen zwischen dem mittleren Drittel des II. Lendenwirbels und der Bandscheibe zwischen dem III. und IV. Lendenwirbel, und geht am häufigsten im kranialen Drittel des III. Lendenwirbels ab. Die Mittelwerte in jeder Altersstufe sind 14.6 bei der I. Gruppe, 14.7 bei der 2. Gruppe, I 5.3 bei der 3. Gruppe und 14.6 bei der 4. Gruppe. Abgesehen von der 4. Gruppe steigt diese Abgangsstelle mit zunehmendem Alter allmählich ab. Die Resultate von den Neugeborenen, Kindern und Erwachsenen tabellarisch zusammengestellt, bringt die Tabelle 8 . Wie aus dieser Tabelle

Tabelle 8. A. mesenterica inf.

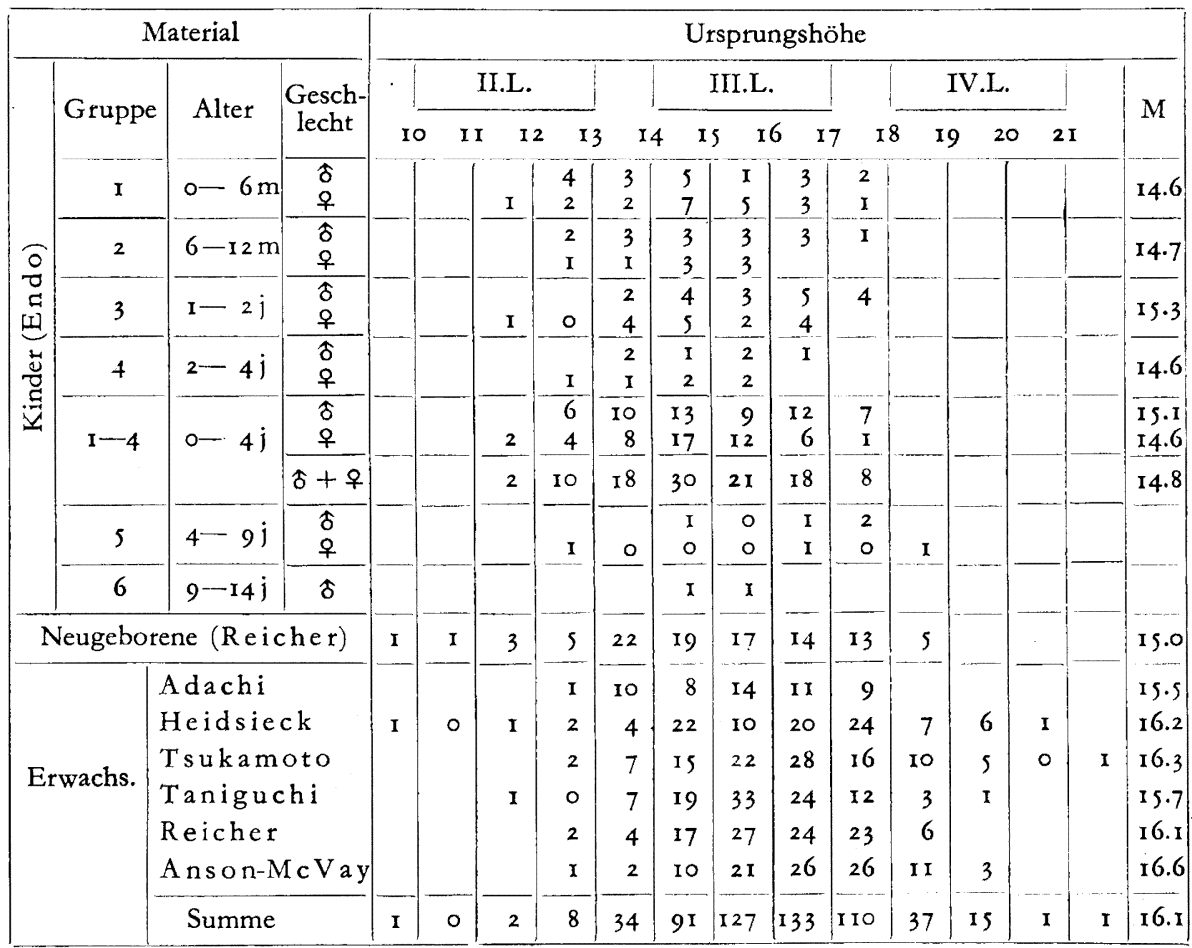

und der Figur 5 ersichtlich ist, ist der Ursprung bei meinem Material durchschnittlich ein wenig höher als bei Neugeborenen und etwas merklich, fast um die Hälfte der Höhe eines Wirbelkörpers, höher als bei Erwachsenen.

Dem Geschlechte nach liegt die Abgangsstelle bei weiblichen Kindern $(M=14.6)$ höher als bei männlichen ( $M=15.1)$. 


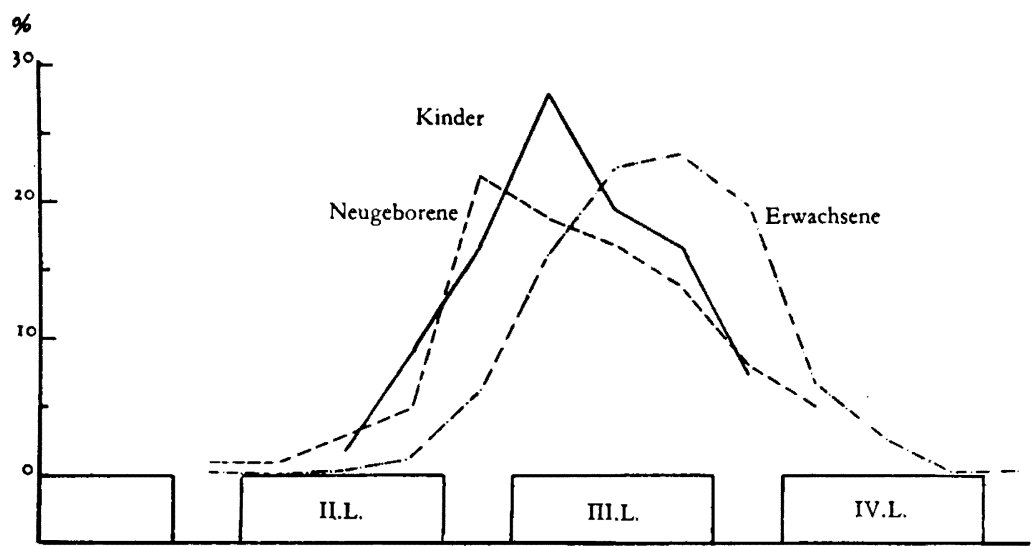

Fig. 5. A. mesenterica inf.

\section{5) Teilungshöhe der Aorta abdominalis gegen die Wirbelsäule.}

Die Höhe der Teilungsstelle der Aorta abdominalis liegt bei meinen Fällen in dem Gebiete vom kaudalen Drittel des III. Lendenwirbels bis zum

Tabelle 9. Höhe der Aortenteilung.

\begin{tabular}{|c|c|c|c|c|c|c|c|c|c|c|c|c|c|c|c|c|c|}
\hline & - & Material & & & & & & & Ursp & prung & gshö & öhe & & & & & \\
\hline & & Altor & Gesch- & & III.L. & & & & IV.L & & & & & V.L. & & & 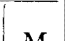 \\
\hline & Grupp & Alter & lecht & I & 16 & $x$ & & 8 & 19 & 20 & $2 \mathrm{I}$ & 22 & 2 & $3 \quad 24$ & 25 & & $\mathrm{M}$ \\
\hline & I & $0-6 \mathrm{~m}$ & $\begin{array}{l}\hat{\delta} \\
\text { \& }\end{array}$ & & & I & 2 & $\begin{array}{l}5 \\
0\end{array}$ & $\begin{array}{l}4 \\
5\end{array}$ & $\begin{array}{l}3 \\
7\end{array}$ & $\begin{array}{l}3 \\
7\end{array}$ & 5 & I & & & & 20.0 \\
\hline & 2 & $6-12 \mathrm{~m}$ & $\delta$ & & & & & 5 & 3 & 3 & 3 & 3 & I & & & & 10.9 \\
\hline$\frac{0}{0}$ & & $0-1210$ & 우 & & - & 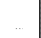 & & 3 & 3 & I & & I & I & & & & 19.9 \\
\hline$\underset{I}{E}$ & 3 & $I-2 j$ & tᄒ & & & $\mathbf{I}$ & $I$ & I & $\begin{array}{l}4 \\
4\end{array}$ & 5 & & $\begin{array}{l}4 \\
2\end{array}$ & 3 & I & & & 20.4 \\
\hline$=$ & & & 南 & & & & 1. & 1 & $\begin{array}{l}4 \\
3\end{array}$ & $\frac{7}{1}$ & & $\begin{array}{l}2 \\
2\end{array}$ & - & & & & $-\infty$ \\
\hline تِّ & 4 & $2-4 j$ & 우 & & & & I & 2 & 2 & $\circ$ & & $\mathrm{I}$ & & & & & I 9.8 \\
\hline 江 & & & $\delta$ & & & & & II & 14 & I 2 & & 14 & 5 & I & & & 20.3 \\
\hline & $\mathrm{I}-4$ & $o-4 j$ & 우 & & $\ldots$ & 2 & 4 & 6 & 14 & Is & & 9 & & & & & 19.8 \\
\hline & & 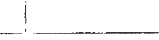 & $5+q$ & & & 2 & 4 & 17 & 28 & 27 & & 23 & 5 & I & & & 20.1 \\
\hline & 5 & $4-9 j$ & $\begin{array}{l}\hat{\delta} \\
8\end{array}$ & & & & & & $\begin{array}{l}I \\
I\end{array}$ & I & & $\begin{array}{l}\circ \\
\circ\end{array}$ & $\begin{array}{l}2 \\
I\end{array}$ & & & & \\
\hline & 6 & $9-14 j$ & $\delta$ & & & & & & & 2 & 2 & & & & & & \\
\hline & eugebo & rene ( $\mathrm{Re}$ ic) & her) & & I & 3 & Is & 25 & I9 & 16 & & is & 7 & & & & 19.5 \\
\hline & & Adachi & & & & & 3 & I 3 & so & 64 & & 46 & 25 & 5 & 2 & & 20.7 \\
\hline & & Heidsieck & & & & & 4 & 10 & Is & 26 & & 27 & 12 & 3 & I & & 20.7 \\
\hline & & Quain & & I & 2 & 3 & I 2 & 43 & 36 & 47 & & 30 & $2 \mathrm{I}$ & o & I & & 19.9 \\
\hline $\mathrm{Er}$ & vachs. & Ssoson-Ja & ro- & & & & & & & & & & & & & & \\
\hline & & schewits & $\mathrm{sch}$ & & I & $\mathbf{I}$ & 5 & 8 & 14 & 15 & & 10 & 14 & I & 2 & & 20.4 \\
\hline & & Tsukamot & & & & & I & I3 & I 2 & 19 & & 26 & 8 & 8 & 8 & 4 & $2 \mathrm{I} \cdot 3$ \\
\hline & & Taniguch & & & & I & $\mathbf{I}$ & 3 & 14 & 42 & & 33 & 3 & 3 & & & 20.7 \\
\hline & & Reicher & & & & & 3 & 15 & 26 & 34 & & 23 & II & 2 & & & 20.4 \\
\hline & & Anson-Mc & Vay & & & I & 3 & 8 & I4 & 32 & & 28 & I0 & 3 & I & & 20.7 \\
\hline & & Summe & & I & 3 & 6 & 32 & $\begin{array}{llll}1 & 1 & 3\end{array}$ & 181 & 279 & 92 & 23 & ro4 & 25 & Is & 4 & 20.5 \\
\hline
\end{tabular}


mittleren Drittel des V. Lendenwirbels; sie liegt am häufigsten im mittleren Teil des IV. Lendenwirbels. Als die Mittelwerte der Teilungshöhe in jeder Altersstufe bekam ich folgende Ziffern : 20.0 bei der x. Gruppe, 19.9 bei der 2. Gruppe, 20.4 bei der 3. Gruppe und 19.8 bei der 4 . Gruppe. So kann man ersehen, dass die Teilungsstelle bei der 3. Gruppe am niedrigsten, und bei der 4. Gruppe am höchsten gelegen sind. In der Tabelle 9 habe ich die Mittelwerte der Teilungshöhe aus den Befunden von Erwachsenen (Adachi, Heidsieck, Quain, Ssoson-Jaroschewitsch, Tsukamoto, Taniguchi, Reicher und Anson-McVay) sowie von Neugeborenen (Reicher) errechnet. Aus dieser Tabelle ersieht man, dass die Teilungsstelle bei Neugeborenen durchschnittlich am höchsten ist, dann folgen die Kinder und bei Erwachsenen ist sie am niedrigsten. Die Differenz der Teilungshöhe zwischen den Kindern und Erwachsenen ist beträchtlich klein, d.h. nur um ein Drittel der Höhe eines Wirbelkörpers, wie die Figur 6 zeigt.

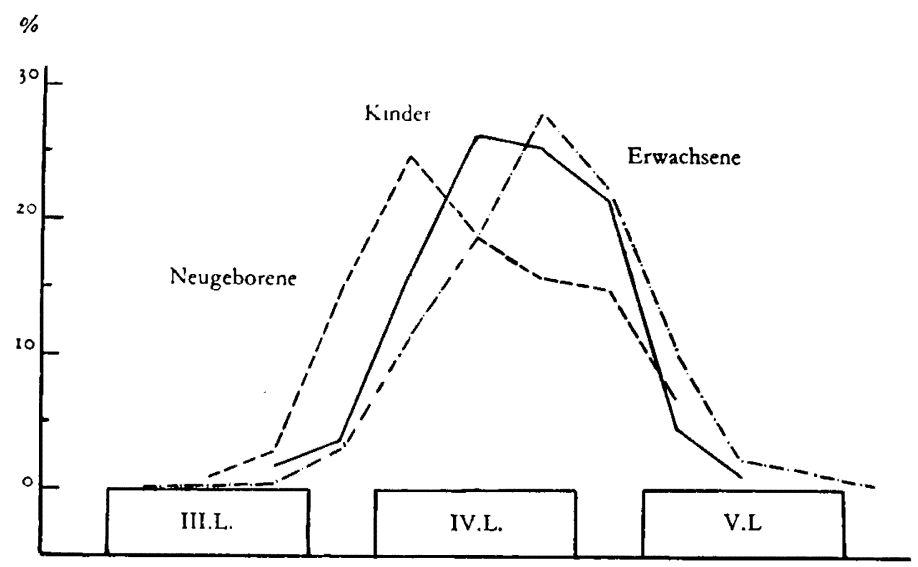

Fig. 6. Höhe der Aortenteilung.

Dem Geschlechte nach liegt sie bei männlichen Kindern $(M=20.3)$ niedriger als bei weiblichen $(M=19.8)$.

\section{Zusammenfassung.}

Ich habe 107 japanische, neugeborene bis 4 jährige Kinder unter besonderer Berücksichtigung des Geschlechts- und Alterunterschiedes untersucht. Im folgenden werde ich die dabei erhaltenen interessanten Befunde kurz zusammenfassen :

I) Ich konnte konstatieren, dass die Abgangsstelle der Bauchaortenäste und die Aortenteilung mit zunehmendem Alter gegen die Wirbelsäule allmäh- 
lich nach kaudal sich verschieben. Der Verschiebungsgrad der Aortenteilung ist am geringsten und der der Abzweigungsstelle der A. coeliaca am grössten. Also kann man mit Recht sagen, dass je ferner das Gefäss von der Aortenteilung ist, umso grösser der Verschiebungsgrad gegen die Wirbelsäule ist, wie die Tabelle ro zeigt.

Tabelle ı. Proportion der Mittelwerte zwischen den

Kindern und Erwachsenen.

\begin{tabular}{|c|l|l|l|l|l|l|}
\hline A. coel. & A. mes. sup. A. ren. dex. & A. ren. sin. & A. mes. inf. & Aortenteil. \\
\hline$\frac{M \text { (Kind. })}{M(\text { Erwachs. })}$ & $\frac{3.6}{6.5}=0.554$ & $\frac{5.5}{8.1}=0.679$ & $\frac{7.4}{9.5}=0.779$ & $\frac{7.8}{9.9}=0.788$ & $\frac{14.9}{16,1}=0.913$ & $\frac{20.1}{20.5}=0.980$ \\
\hline
\end{tabular}

$\mathrm{M}=$ Mittelwert der Ursprungs- und Teilungshöhe.

2) Abgesehen von einigen Ausnahmen, entspringen die oben genannten Gefässe bei älteren Kindern kaudaler als bei jüngeren.

3) Vergleicht man meine Befunde bei der I. Gruppe (Durchschnittsalter von 3.3 Monaten) mit den von R ei cher (Durchschnittsalter von 2 Monaten), so findet man, dass die grossen Bauchaortenäste bei den polnischen Säuglingen etwas niedriger als bei den japanischen entspringen.

4) Was die Ursprungshöhe der beiderseitigen Nierenarterien betrifft, fand ich bei 33 unter I 6 Fällen, also bei $28.45 \%$, den Ursprung beiderseits gleich hoch, in 83 Fällen, also $71.55 \%$ dagegen ungleich hoch, und zwar 57 mal links kaudaler als rechts und 26 mal rechts kaudaler als links, was mit den Befunden der bisherigen Autoren vollständig übereinstimmt.

5) In Bezug auf den Geschlechtsunterschied fand ich die höhere Abzweigung bei den weiblichen als bei den männlichen, worauf andere Autoren schon hingewiesen haben.

Zum Schlusse spreche ich Herrn Professor Dr. S. Mochizuki, Herrn Prof. Dr. T. Tanigu chi und Herrn Assistent-Prof. Dr. S. Kato für ihre stete freundliche Unterstützung bei dieser Arbeit meinen herzlichsten Dank aus.

\section{Literatur.}

I. Adachi, B., Das Arteriensystem der Japaner. Band I und II. Tokyo. 1928.

2. Anson, J. and McVay, B., The topographical positions and the mutual relations of the visceral branches of the abdominal aorta. Anatomical tecord. Vol. 67. 1936.

3. A so, M., Über das Gefässsystem des Rumpfes bei den japanischen Föten. II. Abteilung. Das Arteriensystem. Kaibogaku-Zasshi. Bd. 5. 1932. (Japanisch).

4. Bloch, J. C. et Michon, L., L'origine des grosses branches de l'aorte abdominale. Bulletins et Mémoires de la Société anatomique de Paris. $92^{\mathrm{c}}$ Annèe. 1922. (zitiert bei Adachi). 
5. Broman, I., Über die Entwicklung und „Wanderung“ der Zweige der Aorta abdominalis beim Menschen nebst Bemerkungen über Gefässwurzelwanderungen im allgemeinen. Anat. Hefte. Bd. 36.1908.

6. Broesike, G., Lehrbuch der normalen Anatomie des menschlichen Körpers. Io. Auf. Berlin. I920.

7. Corsy et Aubert, Artéres de L'intestin Grêle et des côlons. Bibliographie anatomique. T. 23. 1913. (zitiert bei Adachi).

8. Dwight, T., The branches of the superior mesenteric artery of the jejunum and ileum. Anat. Anz. Bd. 23. 1903.

9. Frédéric, J., Beitrag zur Anatomie und Entwicklungsgeschichte der Äste der Aorta descendens beim Menschen. Morph. Arbeiten. Bd. 7. 1897.

10. Heidsieck, E., Zur Skeletotopie der grossen Äste der Bauchaorta. Anat. Anz. Bd. 66. 1928.

II. Helm, F., Zur Topographie der menschlichen Nieren. Anat. Anz. Bd. II. I896.

12. I ijima, H., Lageverhalten der A. und V. renalis und des Ureters der Japaner. TokyoIịi-Shinshi. Nr. 24I5. 1909. (Japanisch).

13. Kubo, T., Über die Varietäten der A. renalis. Tokyo-Igakkwai-Zasshi. Bd. 21. 1907. (Japanisch).

14. Levi, G., Le variazioni delle arterie surrenali e renali studiate col metodo statistico seriale. Arch. Ital. Anat. e Embirol. Vol. 8. 1909. (zitiert bei He id s i eck).

I5. Monguidi, C., Topografia dei principali rami viscerali del'Aorta abdominale (con applicazioni alla chirurgia). Milano. I 894 . (zitiert bei Adachi).

16. Nagasawa, Y., Niere und Arterie. Pathol. und Therap. Bd. 1. I927. (Japanisch).

17. Piquand, G., Recherches sur l'anatomie du tronc coeliaque et ses branches. Bibliographie anatomique. T. r9. Igro. Schwalb es Jahresberichte. Bd. I9.

18. Ooniwa, M., Vergleichend-anatomische Bemerkung über die Lage der Niere. KaibogakuZasshi. Bd. 2. 1929. (Japanisch).

19. Qua in, Anatomy of the arteries of the human body. London. 1844. (zitiert bei A da chi).

20. Reicher, M., Topographie der Aorta und der Ursprünge ihrer grossen Bauchäste beim Erwachsenen und beim Neugeborenen. Travaux de la société des sciences et des lettres de Wilno. Classe des Sciences mathématiques et naturelles. Tome VII. 1933.

21. Ssoson-Jaroschewitsch, A. J., Zur chirurgischen Anatomie der Aortabifurkation. Zeitschr. f. Anat. und Entw.-Gesch. Bd. 79. 1926.

22. Taniguchi, T., Beitrag zur Topographie der grossen Äste der Bauchaorta. Folia Anatomica Japonica. Bd. 9. I93I.

23. Tsukamoto, N., Úber die Arterien in der Bauchhöhle bei den Japanern. KaibogakuZasshi. Bd. 2. 1929. (Japanisch). 